\title{
The clinical impact of molecular breast imaging in women with proven invasive breast cancer scheduled for breast-conserving surgery
}

\author{
Angela Collarino ${ }^{1,2}\left(\mathbb{D} \cdot\right.$ Renato A. Valdés Olmos ${ }^{1,3,4} \cdot$ Lotta G. A. J. van Berkel $^{5}$. Peter A. Neijenhuis ${ }^{6}$. \\ Lidy M. H. Wijers ${ }^{5}$. Frederik Smit ${ }^{1,3,7} \cdot$ Lioe-Fee de Geus-Oei ${ }^{1,2} \cdot$ Lenka M. Pereira Arias-Bouda ${ }^{1,7}$
}

Received: 11 September 2017 / Accepted: 3 February 2018 / Published online: 13 February 2018

(C) The Author(s) 2018. This article is an open access publication

\begin{abstract}
Purpose To investigate the clinical utility of molecular breast imaging (MBI) in patients with proven invasive breast cancer scheduled for breast-conserving surgery (BCS).

Methods Following approval by the institutional review board and written informed consent, records of patients with newly diagnosed breast cancer scheduled for BCS who had undergone MBI for local staging in the period from March 2012 till December 2014 were retrospectively reviewed.

Results A total of 287 women (aged 30-88 years) were evaluated. MBI showed T stage migration in 26 patients (9\%), with frequent detection of in situ carcinoma around the tumor. Surgical management was adjusted in 14 of these patients (54\%). In 17 of 287 patients (6\%), MBI revealed 21 proven additional lesions in the ipsilateral, contralateral breast or both. In 18 of these additional foci (86\%), detected in 15 patients, malignancy was found. Thirteen of these 15 patients had ipsilateral cancer and 2 patients bilateral malignancy. In total, MBI revealed a larger tumor extent, additional tumor foci or both in 40 patients (14\%), leading to treatment adjustment in 25 patients (9\%).

Conclusion MBI seems to be a useful imaging modality with a high predictive value in revealing ipsilateral and bilateral disease not visualized by mammography and ultrasound. It may play an important role in delineating the extent of the index lesion during preoperative planning. Incorporation of MBI in the clinical work-up as an adjunct modality to mammography and ultrasound may lead to better selection of patients who could benefit from BCS.
\end{abstract}

Keywords Breast cancer · Preoperative breast imaging · Molecular breast imaging · MBI - Breast-specific gamma imaging BSGI

Angela Collarino

angelacollarino@tiscali.it

1 Section of Nuclear Medicine, Department of Radiology, Leiden University Medical Center, Albinusdreef 2, 2333 ZA Leiden, The Netherlands

2 Biomedical Photonic Imaging Group, MIRA Institute, University of Twente, 217, 7500 AE Enschede, The Netherlands

3 Interventional Molecular Imaging Laboratory, Department of Radiology, Leiden University Medical Center, Albinusdreef 2, 2333 ZA Leiden, The Netherlands
4 Department of Nuclear Medicine, The Netherlands Cancer Institute-Antoni Van Leeuwenhoek Hospital, Plesmanlaan 121, 1066 CX Amsterdam, The Netherlands

5 Department of Radiology, Alrijne Ziekenhuis, Simon Smitweg 1, 2353 GA Leiderdorp, The Netherlands

6 Department of Surgery, Alrijne Ziekenhuis, Simon Smitweg 1, 2353 GA Leiderdorp, The Netherlands

7 Department of Nuclear Medicine, Alrijne Ziekenhuis, Simon Smitweg 1, 2353 GA Leiderdorp, The Netherlands 


\section{Introduction}

Invasive breast cancer is the most common cancer among women, with an incidence of 14.479 new cases in 2016 in The Netherlands [1] and 1.67 million new cancer cases diagnosed in 2012 worldwide [2].

In the last decades, breast-conserving surgery (BCS), also called lumpectomy, has gained importance due to the possibility to remove the tumor preserving the natural shape of the breast [3]. BCS is contraindicated in small breasts with large primary tumors and in case of multicentric tumors [3, 4]. Therefore, accurate definition of the extent of the primary tumor and exclusion of additional foci of cancer (multifocal, multicentric and contralateral breast cancer) is important in order to conduct the appropriate surgical treatment. Currently, magnetic resonance imaging (MRI) and molecular breast imaging (MBI) have been indicated to assess tumor extent and multifocal, multicentric and contralateral disease in adjunction to mammography (MG) and ultrasonography (US) [5, 6]. MBI is a functional imaging technique consisting of a breastdedicated gamma camera equipped with a small fieldof-view (FOV) single- or dual-head detector, producing high-resolution images corresponding to the standard projections used in MG [7-11]. In MBI, tumor-seeking radiopharmaceuticals like ${ }^{99 \mathrm{~m}} \mathrm{Tc}$-sestamibi are used. Uptake of this tracer into tumor cells is based on increased vascularity and high mitochondrial density [12-14]. Recently, a low-dose protocol with an injected dose of $260-500 \mathrm{MBq}$ ${ }^{99 \mathrm{~m}} \mathrm{Tc}$-sestamibi has been introduced using a single-head MBI device $[15,16]$. Dual-head MBI devices allow even lower injected doses varying from 150 to $300 \mathrm{MBq}$ of ${ }^{99 \mathrm{~m}} \mathrm{Tc}$-sestamibi $[11,17]$. This leads to both reduction of absorbed dose and effective dose to the breast [18]. Compared to MRI, MBI is easy to interpret, is associated with low costs and is not contraindicated in patients with claustrophobia, overweight, implanted devices and renal insufficiency.

The purpose of this study was to investigate the clinical utility of MBI in adjunction to MG and US for delineation of the extent of the index lesion and to rule out additional tumor foci in patients with invasive breast cancer scheduled for BCS.

\section{Materials and methods}

\section{Patients}

The institutional review board approved this retrospective study and informed consent was obtained from all patients.
Patients were included if they fulfilled the following criteria: (a) presence of histopathologically proven invasive breast cancer; (b) after conventional clinical work-up (including 2D MG, Siemens Inspiration Mammomat, and 2D US, Philips Affiniti 70 G Linear transducer L 12-5) the patient was scheduled for BCS; (c) the patient had undergone pretreatment MBI for assessment of tumor extent and presence of multifocal or multicentric disease; (d) complete individual data were available concerning clinical work-up, imaging, surgery and histopathology.

\section{MBI acquisition}

MBI imaging was performed using a dedicated device equipped with a single detector system also known as breast-specific gamma imaging (BSGI; Dilon 6800, Dilon Diagnostics, Newport News, Virginia, U.S.A.). Images were acquired with the patient in seated position and with the breast in light compression. At our institution, we used a relative low-dose protocol $(600 \mathrm{MBq})$ in comparison with the most published articles (740-1110 MBq) [7-9]. As mentioned earlier, recent studies showed that it seems possible to use even lower injected doses with BSGI $[15,16]$. Each patient received an injection of approximately $600 \mathrm{MBq}$ of ${ }^{99 \mathrm{~m}} \mathrm{Tc}$-sestamibi into an antecubital vein contralateral to the breast lesion. Approximately 5-10 min after the injection, craniocaudal (CC) and mediolateral oblique (MLO) planar images were obtained for each breast, comparable with those of MG. The acquisition time for each image was 8-10 min, giving a total acquisition time of approximately $40 \mathrm{~min}$ per study. If relevant, additional planar images (lateromedial or mediolateral view, anteroposterior view (axilla) or axillary craniocaudal view) were acquired from the ipsilateral breast.

\section{MBI image analysis}

All MBI images were evaluated by two nuclear medicine physicians of our institute (L.M.P.A-B and F.S.) and were directly compared with the most recent MG following the functional Breast Imaging Reporting and Data System (BIRADS) classification $[6,19]$.

The size of the index lesion was calculated by measuring the maximum diameter $(\mathrm{mm})$ of the pathological uptake on the MBI images ( ${ }_{\mathrm{MBI}} \mathrm{T}$ stage). In case of more than one lesion, the maximum diameter of the largest tumor was used. Index lesion size detected on MBI was compared with the lesion size obtained with MG and US (MORPHOLOGICAL $\mathrm{T}$ stage).

MBI-detected abnormalities were considered to be additional tumor lesions when they were suspicious on MBI (BIRADS 4 or 5) and occult on MG and initially not picked up on US. At our institute, US is used to characterize a palpable mass or to find a correlate for a mammographical lesion. According 
to this criterion, the radiologist performed US of a sole lesion and not of a quadrant of the breast nor the whole breast. The additional breast lesions were classified as follows: (1) multifocal lesions when located in the same quadrant of the breast as the index tumor; (2) multicentric lesions when located in a different quadrant of the breast compared to the index tumor; and (3) contralateral lesions when located in the contralateral breast. The size of each additional lesion was measured on MBI corresponding to the maximum diameter $(\mathrm{mm})$ of the pathological uptake. Histopathology was obtained from all additional MBI lesions after incisional needle-biopsy or surgical excision. The biopsy was performed using US-guided biopsy when the lesion was visible on targeted US. In more detail, after performing MBI and finding an additional suspicious lesion, the patient returned to the radiology department to undergo targeted US. In most cases, the additional detected lesion was previously not picked up during diagnostic workup, since no routine whole breast screening US was performed. Targeted US was performed directly after MBI in case of an unexpected additional lesion, followed by US-guided biopsy. The radioactivity in the biopsy specimen was measured to prove that the lesion found on targeted US corresponded to the additional lesion found with MBI. In case of additional BI-RADS $4 \mathrm{~b}, \mathrm{c}$ or 5 lesions on MBI that remained occult on targeted US, MBI-guided biopsy was performed, but only if clinically relevant. BI-RADS 4a MBI abnormalities where considered benign if no correlate was found at targeted US.

\section{Statistical analysis}

The $\chi^{2}$ test was used to analyze significant differences between dense and non-dense index lesion tumor as well as high-grade and low-grade. A $p$ value of $<0.05$ was considered statistically significant. Statistical analysis was performed using MedCalc Statistical Software version 15.11.4.

Based on T stage migration (upstaging) after MBI, the percentage of patients in who surgical management was adjusted based on the MBI results was calculated. Based on the biopsy-or excision-acquired pathological findings, all additional lesions with malignant histopathology like invasive tumor and ductal carcinoma in situ (DCIS) were considered true positive, while all additional lesions with benign histopathology were defined false positive. On the basis of the detected additional lesions on MBI, the lesion-based positive predictive value (PPV) was calculated using the formula True-positive/True-positive + False Positive $\times 100$.

\section{Results}

Records of 304 women with proven invasive breast cancer scheduled for BCS who underwent MBI between March 2012 and December 2014 were reviewed. Seventeen of these women, who had additional MBI-detected lesions without histopathological diagnosis, were excluded from the final analysis. In more detail, in 4 of these 17 patients the multidisciplinary team agreed upon that it was not necessary to prove the malignant nature of the lesion, because it was located nearby the index lesion and would not alter the treatment plan. Since these patients were treated with neoadjuvant therapy, it was not possible to verify the nature of the additional lesion afterwards. Thirteen of 17 patients had focal MBI lesions classified as BI-RADS 4a, meaning

Table 1 Title: Patient characteristics

\begin{tabular}{|c|c|}
\hline Number of pts & 287 \\
\hline Mean age (range) & $60(30-88)$ \\
\hline \multicolumn{2}{|l|}{ Menopausal status } \\
\hline Pre-/perimenopausal & 79 \\
\hline Postmenopausal & 208 \\
\hline \multicolumn{2}{|l|}{ Breast tissue composition* } \\
\hline $\mathrm{a}$ & 35 \\
\hline $\mathrm{b}$ & 107 \\
\hline $\mathrm{c}$ & 127 \\
\hline $\mathrm{d}$ & 18 \\
\hline Mean tumor size (range) & $18 \mathrm{~mm}(3-55 \mathrm{~mm})$ \\
\hline Multifocal/multicentric & 18 \\
\hline \multicolumn{2}{|l|}{ T Stage prior to surgery } \\
\hline T1a & 6 \\
\hline $\mathrm{T} 1 \mathrm{~b}$ & 63 \\
\hline $\mathrm{T} 1 \mathrm{c}$ & 128 \\
\hline $\mathrm{T} 2$ & 89 \\
\hline Unknown & 1 \\
\hline \multicolumn{2}{|l|}{ Tumor type } \\
\hline IDC & 246 \\
\hline ILC & 24 \\
\hline Mixed IDC/ILC & 1 \\
\hline Other & 16 \\
\hline \multicolumn{2}{|l|}{ Tumor subtype } \\
\hline HER2-positive & 40 \\
\hline ER-positive/HER2-negative & 199 \\
\hline Triple negative & 38 \\
\hline \multicolumn{2}{|c|}{ Scarff-Bloom Richardson Grade*** } \\
\hline 1 & 43 \\
\hline 2 & 112 \\
\hline 3 & 124 \\
\hline Unknown & 8 \\
\hline
\end{tabular}

*No significant difference between dense (c, d) and non-dense (a, b) breast tissue $(p=0.8)$

**Significant difference between high-grade (grade 3 ) and low-grade (grade 1,2$)$ breast tumors $(p<0.008)$

a almost entirely fat; ${ }^{b}$ scattered fibroglandular density; ${ }^{c}$ heterogeneously dense; dextremely dense; ${ }^{\mathrm{IDC}}$ invasive ductal carcinoma; ${ }^{\mathrm{ILC}} \mathrm{in}$ vasive lobular carcinoma; ${ }^{\text {DCIS }}$ ductal carcinoma in situ; ${ }^{E R}$ estrogen receptor; ${ }^{\mathrm{HER} 2}$ human epidermal growth factor receptor 2 
Table $2 \mathrm{~T}$ stage migration and treatment adjustment following MBI

\begin{tabular}{|c|c|c|c|c|c|}
\hline $\mathrm{N}$ of pts & $\begin{array}{l}\text { Morphologi- } \\
\text { cal size }(\mathrm{mm})\end{array}$ & MBI size $(\mathrm{mm})$ & $\mathrm{T}$ stage migration & $\begin{array}{l}\text { Treatment } \\
\text { plan before } \\
\text { MBI }\end{array}$ & Treatment plan after MBI \\
\hline 1 & 20 & 24 & $\mathrm{~T} 1>\mathrm{T} 2$ & BCS & $\mathrm{BCS}$ \\
\hline 2 & 20 & 24 & $\mathrm{~T} 1>\mathrm{T} 2$ & $\mathrm{BCS}$ & $\mathrm{BCS}$ \\
\hline 3 & 16 & 35 & $\mathrm{~T} 1>\mathrm{T} 2$ & $\mathrm{BCS}$ & Large BCS \\
\hline 4 & 17 & 30 & $\mathrm{~T} 1>\mathrm{T} 2$ & BCS & BCS \\
\hline 5 & 11 & 35 & $\mathrm{~T} 1>\mathrm{T} 2$ & $\mathrm{BCS}$ & Large BCS \\
\hline 6 & 50 & 55 & $\mathrm{~T} 2>\mathrm{T} 3$ & $\mathrm{NAC}+\mathrm{BCS}$ & $\mathrm{NAC}+\mathrm{BCS}$ \\
\hline 7 & 40 & 63 & $\mathrm{~T} 2>\mathrm{T} 3$ & $\mathrm{NAC}+\mathrm{BCS}$ & $\mathrm{NAC}+\mathrm{BCS}$ \\
\hline 8 & 19 & 40 & $\mathrm{~T} 1>\mathrm{T} 2$ & BCS & Large BCS \\
\hline 9 & 20 & 27 & $\mathrm{~T} 1>\mathrm{T} 2$ & $\mathrm{BCS}$ & Mastectomy \\
\hline 10 & 19 & 30 & $\mathrm{~T} 1>\mathrm{T} 2$ & BCS & Large BCS \\
\hline 11 & 17 & 30 & $\mathrm{~T} 1>\mathrm{T} 2$ & $\mathrm{BCS}$ & Large BCS \\
\hline 12 & 20 & 42 & $\mathrm{~T} 1>\mathrm{T} 2$ & $\mathrm{BCS}$ & $\mathrm{NAC}+\mathrm{BCS}$ \\
\hline 13 & 10 & 85 & $\mathrm{~T} 1>\mathrm{T} 3$ & $\mathrm{BCS}$ & Mastectomy \\
\hline \multirow[t]{2}{*}{14} & 23 & 26 & $\mathrm{~T} 1>\mathrm{T} 2$ & Left BCS & Mastectomy \\
\hline & 13 & 80 & $\mathrm{~T} 1>\mathrm{T} 3$ & Right BCS & Mastectomy \\
\hline 15 & 15 & 21 & $\mathrm{~T} 1>\mathrm{T} 2$ & BCS & BCS \\
\hline 16 & 20 & 32 & $\mathrm{~T} 1>\mathrm{T} 2$ & $\mathrm{BCS}$ & $\mathrm{BCS}$ \\
\hline 17 & 20 & 24 & $\mathrm{~T} 1>\mathrm{T} 2$ & BCS & $\mathrm{BCS}$ \\
\hline 18 & 10 & 26 & $\mathrm{~T} 1>\mathrm{T} 2$ & $\mathrm{BCS}$ & $\mathrm{BCS}$ \\
\hline 19 & 7 & 30 & $\mathrm{~T} 1>\mathrm{T} 2$ & BCS & Mastectomy \\
\hline 20 & 20 & 25 & $\mathrm{~T} 1>\mathrm{T} 2$ & BCS & BCS \\
\hline 21 & 40 & 120 & $\mathrm{~T} 2>\mathrm{T} 3$ & BCS & $\mathrm{NAC}+\mathrm{BCS}$ \\
\hline 22 & 40 & 90 & $\mathrm{~T} 2>\mathrm{T} 3$ & BCS & Quadrantectomy \\
\hline 23 & 40 & 90 & $\mathrm{~T} 2>\mathrm{T} 3$ & BCS & Mastectomy \\
\hline 24 & 19 & 23 & $\mathrm{~T} 1>\mathrm{T} 2$ & BCS & BCS \\
\hline 25 & 16 & 23 & $\mathrm{~T} 1>\mathrm{T} 2$ & BCS & BCS \\
\hline 26 & 16 & 90 & $\mathrm{~T} 1>\mathrm{T} 3$ & BCS & Mastectomy \\
\hline
\end{tabular}

$N$ number, Pts patients, $M B I$ molecular breast imaging, $B C S$ breast-conserving surgery, $N A C$ neoadjuvant chemotherapy that there was doubt about the real nature of the MBI finding, for example because it was visible in only one view and could be caused by over-projection or an artifact. In these 13 patients, the finding was considered benign because no correlate was found with targeted US. The remaining 287 patients who fulfilled the inclusion criteria were enrolled in this retrospective study. The characteristics of the patients are reported in Table 1 . The mean age of the patients was 60 years (range, $30-88$ years). A significant difference was found between high-grade and low-grade breast tumors $(p<0.008)$, since more patients had low-grade tumors (grade 1 or 2). No significant difference was found between dense and non-dense breast tissue $(p=0.8)$ in this study population. The mean morphological maximum tumor diameter, obtained with MG and US, was $18 \mathrm{~mm}$ (range, 3-55 mm). In 246 patients $(86 \%)$, the index lesion concerned invasive ductal carcinoma (IDC), in 24 patients (8\%) invasive lobular carcinoma (ILC), and in 1 patient $(0.3 \%)$ mixed IDC and ILC. The remaining 16 patients (5.7\%) had other tumor types including 6 mucinous carcinomas, 3 papillary carcinomas, 3 apocrine carcinomas, 2 medullary carcinomas, and 2 tubular carcinomas.

Concerning the diameter of the index lesion, concordance between MBI and radiologic imaging was found in 261 patients (91\%). In 26 out of 287 patients (9\%), MBI showed $\mathrm{T}$ stage migration with adjustment of the surgical management in 14 of these 26 patients (54\%) (Table 2). Five patients underwent unilateral mastectomy, 1 patient bilateral mastectomy, another 5 patients were treated with large lumpectomy, 2 patients received NAC before BCS, and 1 patient underwent quadrantectomy. In 10 of these 14 patients, the larger tumor extent on MBI was related to histopathologically proven DCIS around the invasive lesion (Fig. 1).

In 17 of 287 patients (6\%), MBI revealed 21 proven additional lesions in the ipsilateral breast, contralateral breast or both breasts (Table 3). The median size of these lesions on MBI was $10 \mathrm{~mm}$ (range: 7-35 mm). Histopathological features were obtained by needle-biopsy from 16 out of 
21 lesions in 12 patients and by surgical excision from the other 5 lesions concerning another 5 patients. Breast needlebiopsies were performed under guidance of US in 10/12 patients and under MBI guidance in 2/12 patients. In 15 out of these 17 patients $(88 \%)$ the 18 additional lesions turned out to be malignant. In 13 of these 15 patients, the malignant lesions concerned ipsilateral tumors (15/18 lesions) and in 2 patients bilateral tumors (3/18 lesions) (Fig. 2). The surgical management was adjusted in 12 out of these 15 patients (80\%). In more detail, 3 patients were converted to NAC and mastectomy, while 7 patients underwent ipsilateral mastectomy instead of BCS, 1 patient was treated with bilateral mastectomy, and 1 patient with bilateral BCS. From the 18 additional proven malignant lesions (true positives) on MBI, 6 lesions were smaller than $10 \mathrm{~mm}$ (range: $6-8 \mathrm{~mm}$ ). The pathologic findings of the 18 tumors included 10 IDC, 3 ILC, and 5 DCIS. The remaining 3 additionally detected lesions on MBI were benign lesions (false positives) revealing mastopathy in one, one with fibroadenoma, and one with a mixed pattern of mastopathy and adenosis. The MBI lesion-related PPV was $86 \%$.

Overall, MBI showed an unexpected larger tumor extent, additional tumor foci or both in 40 of 287 included patients (14\%). In one of these 40 patients, MBI revealed both a larger index tumor as well as multicentricity (patient $\mathrm{n}^{\circ} 19 \mathrm{in}$ Table 2 and $\mathrm{n}^{\circ} 10$ in Table 3). In 4 of these 40 patients (10\%), the index lesion concerned ILC. Twenty patients (50\%) had non-dense breast tissue with breast composition a in 4 and breast composition $b$ in 16 patients. Owing to the use of MBI, the overall treatment was adjusted in 25 patients (9\%) (Fig. 3).

\section{Discussion}

In the present study, we evaluated the clinical impact of ${ }^{99 \mathrm{~m}} \mathrm{Tc}$-sestamibi MBI, incorporated in the diagnostic workup of patients with newly diagnosed breast cancer scheduled for BCS, as an adjunct modality to MG and US. Based on our data, the preoperative use of MBI in this specific patient population resulted in the detection of a significantly larger disease extent, additional tumor foci or both in $14 \%$ of patients, albeit $50 \%$ of these patients had non-dense breast tissue on MG and only $10 \%$ had lobular type of carcinoma. The unexpected MBI findings led to treatment adjustment in $9 \%$ of all patients.

Evaluation of the extent of the index lesion using MBI showed a change of the local stage in 26 patients $(9 \%)$ and local excision was abandoned in 14 of these patients. In our series, a larger disease extent as detected on MBI was mainly due to the visualization of DCIS located around the invasive tumor, which is in concordance with the findings of Spanu et al. [20]. Interestingly, in the majority of these patients no calcifications were found in the DCIS area on MG. Although it is not possible to distinguish carcinoma in situ from invasive tumor based on the ${ }^{99 \mathrm{~m}} \mathrm{Tc}$-sestamibi uptake pattern, the total area of pathological ${ }^{99 \mathrm{~m}} \mathrm{Tc}$-sestamibi uptake guided our surgeons during the surgical procedure, increasing the rate of complete surgical treatment and avoiding additional surgeries. Therefore, we postulate that MBI offers the possibility to plan resection of the index lesion more accurately based on the extension of ${ }^{99 \mathrm{~m}} \mathrm{Tc}$-sestamibi uptake.

Additional lesions were visualized on MBI in 17 women (6\%). Two of these patients underwent MBI-guided biopsy since the additional lesions remained occult even after targeted US. MBI-guided biopsy is a biopsy modality approved by the U.S. Food and Drug Administration (FDA) in 2009. This tool is based on stereotactic localization of the
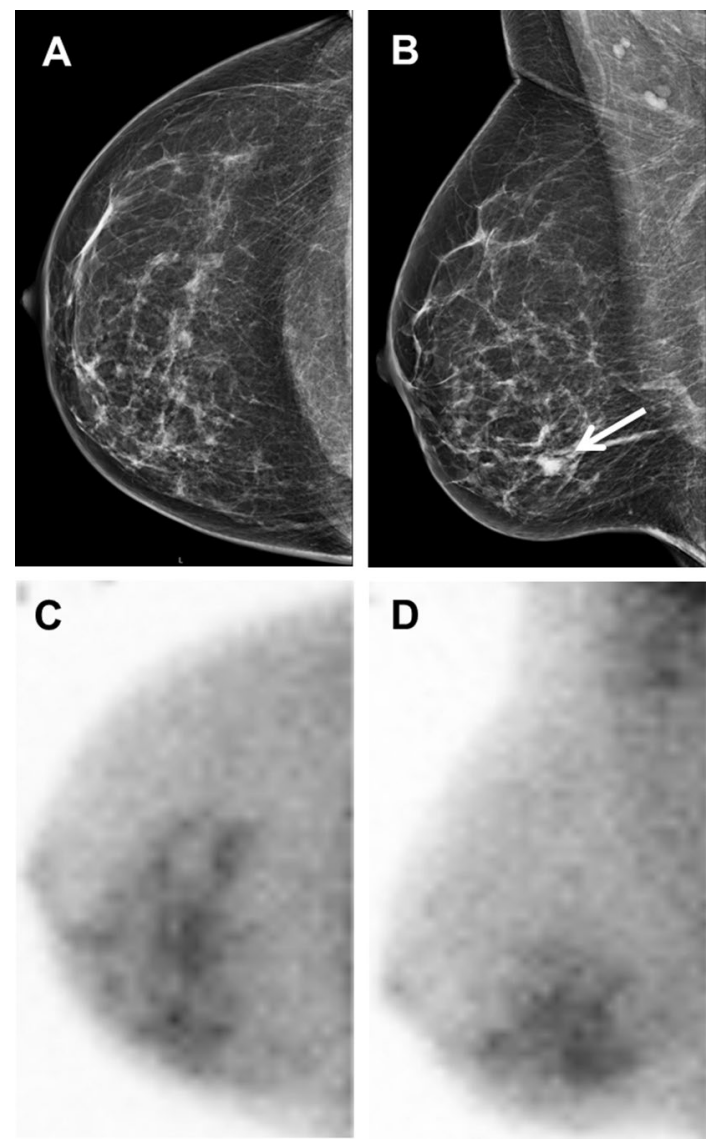

Fig. 1 A 52-year-old woman (patient 13, Table 2) with invasive breast cancer. a Right craniocaudal mammographic image and b right mediolateral oblique mammographic image showing a breast mass of $10 \mathrm{~mm}$ with new calcifications in the lower inner quadrant of the right breast, best visible on the mediolateral oblique view (white arrow). c Right craniocaudal and $\mathbf{d}$ right mediolateral oblique MBI images showing a large and heterogenous area of pathological uptake $(85 \mathrm{~mm})$ in the lower inner quadrant of the right breast. The treatment changed from lumpectomy to mastectomy. Pathological findings revealed intracystic papillary adenocarcinoma and extralesional ductal carcinoma in situ with extension towards the nipple 


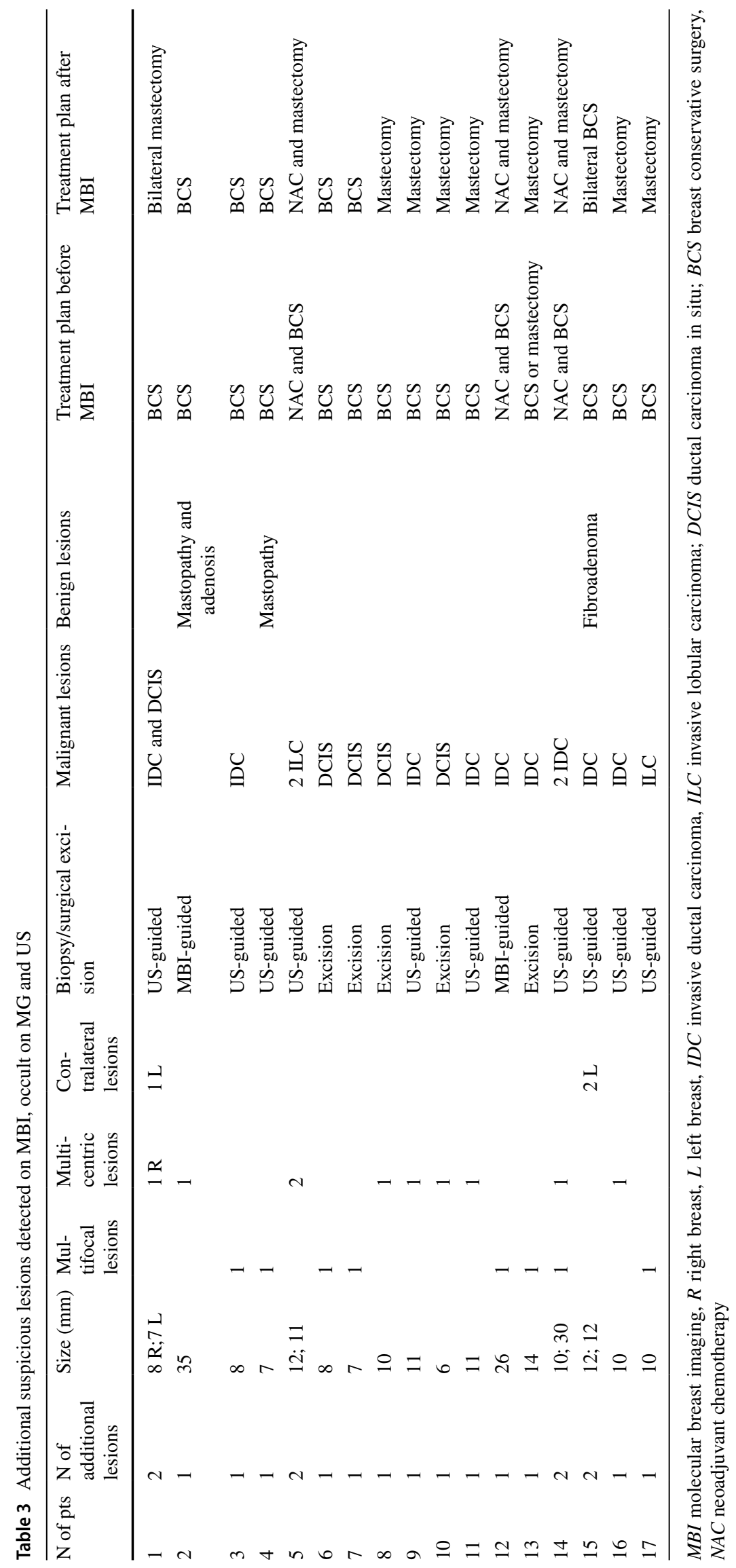



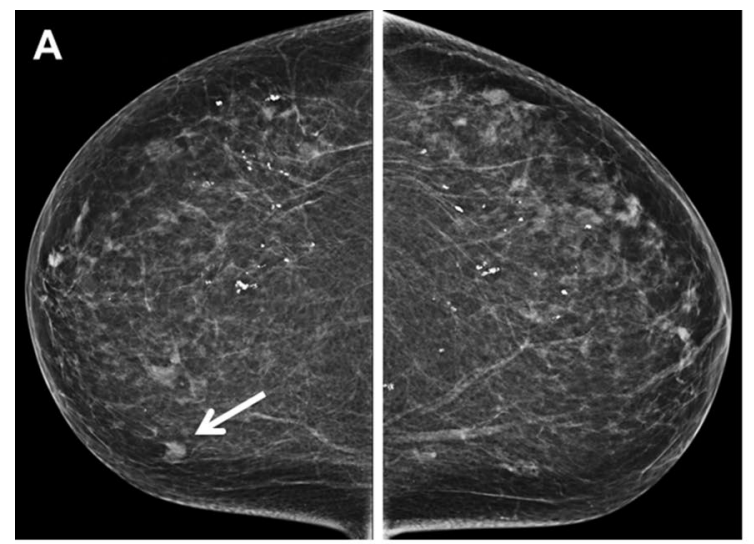

C
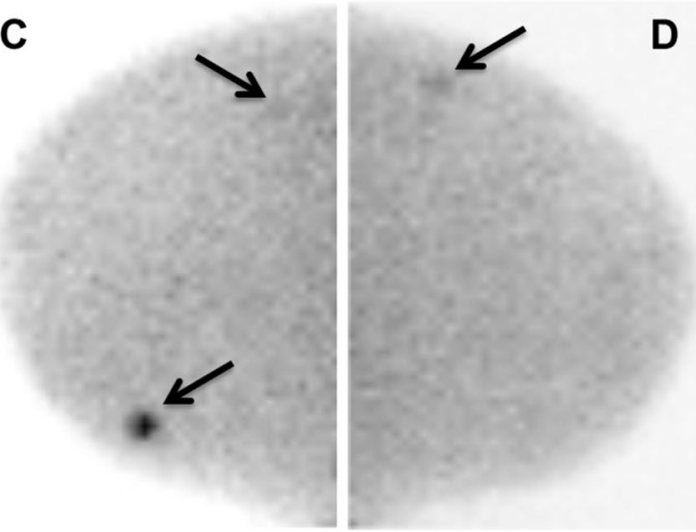

Fig. 2 A 71-year-old woman (patient 1, Table 3) with invasive ductal carcinoma of the right breast and two additional tumor foci (1 in ipsilateral breast and 1 in contralateral breast). a Right craniocaudal and b right mediolateral oblique mammographic images showing a mass of $9 \mathrm{~mm}$ in the lower inner quadrant of the breast (white arrows). c Right craniocaudal MBI image showing two foci with pathological ${ }^{99 \mathrm{~m}} \mathrm{Tc}$-sestamibi uptake (arrows), one intense accumulation medially corresponding to the mass seen on mammography and a mild accumulation laterally corresponding to the new 8-mm lesion located in the upper outer quadrant (multicentric lesion). d Left craniocaudal MBI image shows a new mild focal accumulation in the upper outer quadrant (arrow). e Right mediolateral oblique MBI image shows

${ }^{99 \mathrm{~m}} \mathrm{Tc}$-sestamibi avid lesion [21, 22] and is currently used in our clinical work-up [23]. In our series, MBI-detected lesions corresponded to additional proven tumors in 15 women ( $5 \%$ overall detection rate). This is in line with the results reported in previous studies in the literature [20, 24-29]. Lesion-related analysis demonstrated that 18 out of 21 additional lesions visualized on MBI resulted in true cancer. The high PPV of $86 \%$ suggests that a positive MBI scan is highly predictive for occult tumor. This is in concordance with the relative high specificity of this technique as described in the literature [30]. A possible explanation is the highly specific uptake of ${ }^{99 \mathrm{~m}} \mathrm{Tc}$-sestamibi by tumor cells as compared to the surrounding breast tissue [12-14]. Moreover, MBI detected 6 subcentimeter additional cancers in our
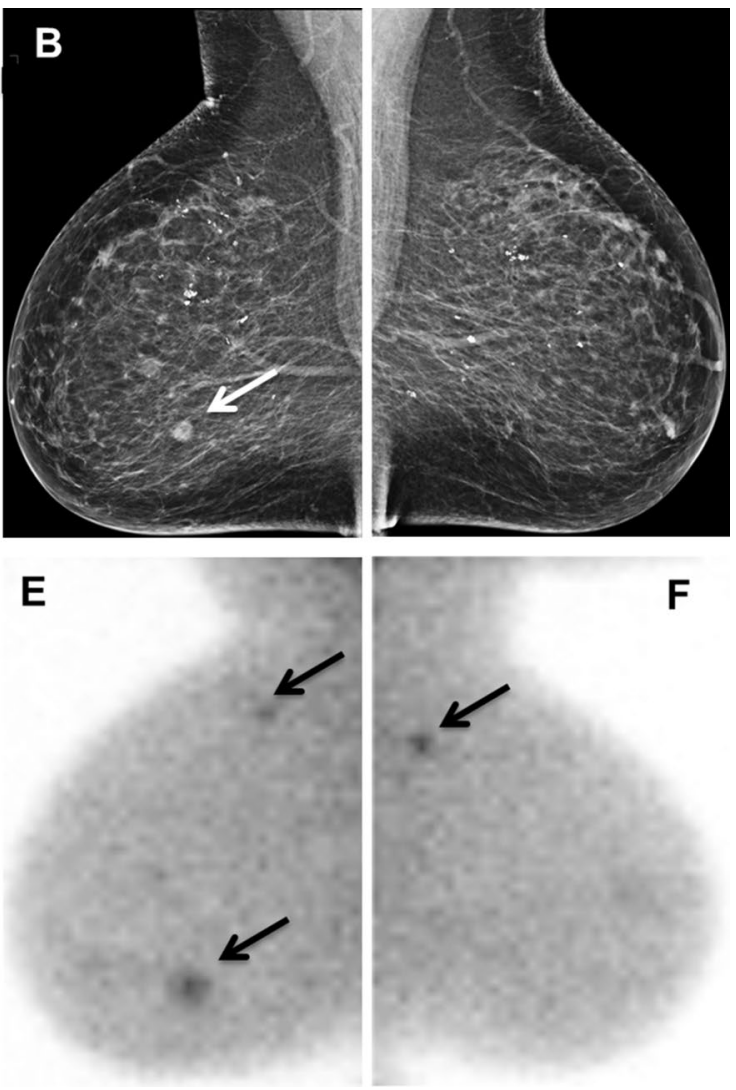

two intense foci (arrows): a caudal accumulation corresponding to the mass seen on mammography and a cranial accumulation $(8 \mathrm{~mm})$ corresponding to the new lesion located in the upper outer quadrant (multicentric lesion). f Left mediolateral oblique MBI image showing a focal intense accumulation of $7 \mathrm{~mm}$ in the upper outer quadrant (contralateral lesion). For both additional lesions, the patient underwent US-guided biopsy after targeted US that revealed invasive ductal carcinoma in the additional lesion in the right breast and ductal carcinoma in situ in the additional lesion in the left breast. The treatment changed from local excision (right breast) to bilateral mastectomy

series. This agrees with prior studies $[20,27,28]$ reporting the ability of MBI to identify occult tumors smaller than $1 \mathrm{~cm}$. Recently, new MBI systems based on dual-head cadmium-zinc-telluride (CZT) detectors have been introduced offering improved sensitivity for detection of small tumors [11]. In our series the detection of additional tumors on MBI has led to the abandonment of lumpectomy in 11 women. Combining the contribution of MBI in relation to investigating disease extent and presence of multifocal and multicentric disease, the overall management was adjusted in $9 \%$ of all patients with newly diagnosed BC scheduled for lumpectomy. Additionally, MBI showed a low false-positive rate, thus avoiding unnecessary biopsies, complementary imaging, and patient anxiety. 


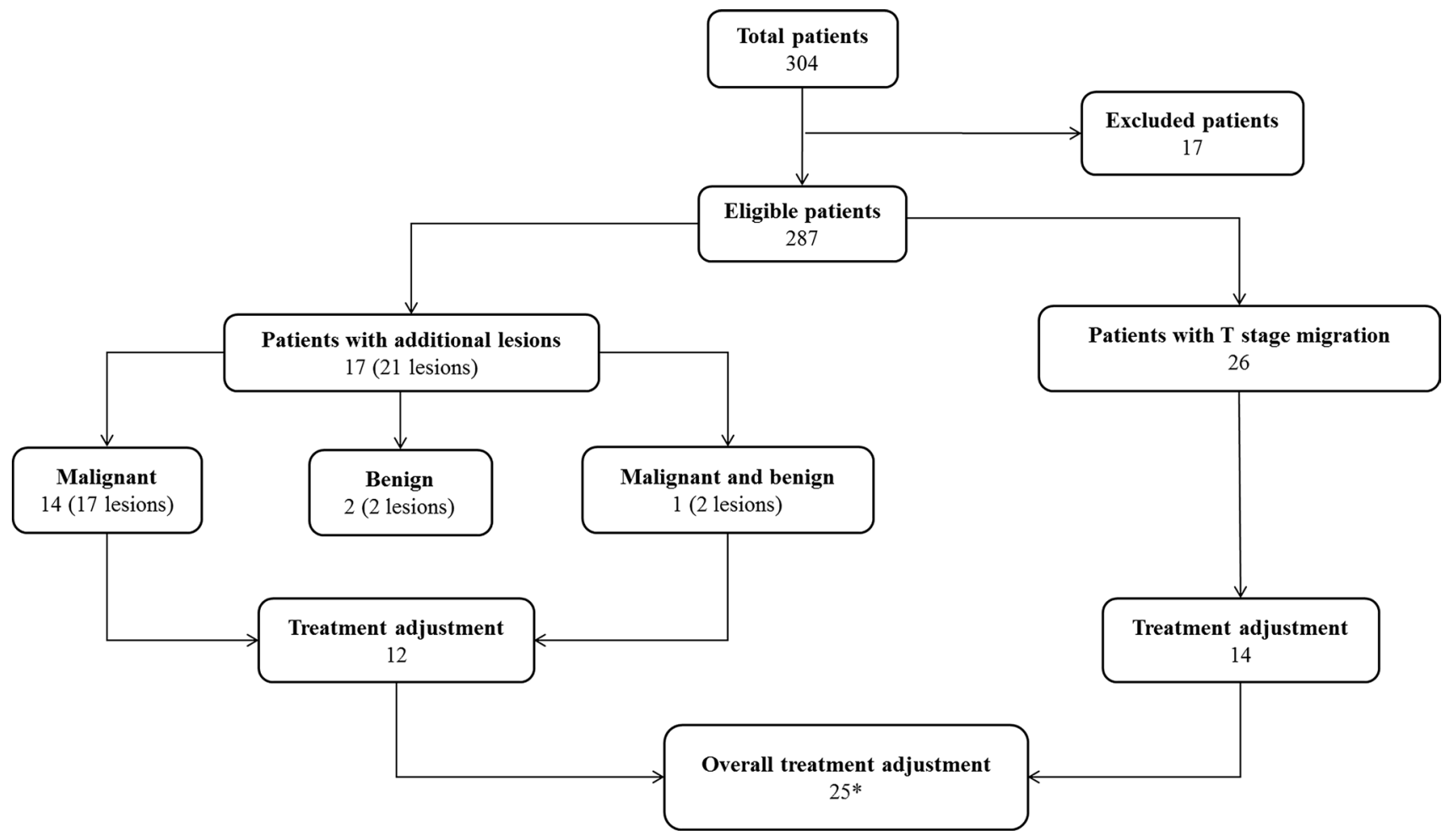

Fig. 3 Flowchart showing the impact of preoperative MBI in the study population. *One patient had both $\mathrm{T}$ stage migration as well as an additional malignant lesion on MBI

In the light of our results, MBI could be a valid adjunct modality to MG and US for detecting both the extent of index lesions and additional tumor foci. Presently, MRI is widely used in the clinical work-up of newly diagnosed breast cancer in women. Although MRI shows high sensitivity, its low specificity and high costs limit a wide application of this modality. Additionally, MRI is not applicable in patients with claustrophobia, overweight, implanted devices and renal insufficiency [31]. MBI has the potential to overcome these limitations becoming a useful tool for almost all newly diagnosed breast cancer patients. Additionally, MBI is easy to perform and is associated with low costs. On the other hand, MBI requires the intravenous injection of a radiotracer, like ${ }^{99 \mathrm{~m}} \mathrm{Tc}$-sestamibi, which means radiation exposure for the patient. However, one should keep in mind that the administered dose of ${ }^{99 \mathrm{~m}} \mathrm{Tc}$-labeled sestamibi for MBI is similar to the dose used for other commonly applied diagnostic functional imaging examinations such as bone scintigraphy and myocardial perfusion imaging [32,33]. Moreover, recent technological advances in MBI allow a significant reduction of the injected dose of the radiopharmaceutical. Indeed, it is possible to use a low-dose imaging protocol with CZT-based dual-head MBI (150-300 MBq) [11, 17] as well as with NaI-based single-head MBI (260-500 MBq) $[15,16]$. An administered dose of $150 \mathrm{MBq}{ }^{99 \mathrm{~m}} \mathrm{Tc}$-sestamibi leads to a significant reduction of absorbed dose to the breast (0.25 mGy) and effective dose (1.1 mSv) [18].

Finally, it is necessary to address the principal limitations of the present study. First, it concerns a retrospective study based on data collected in a single institution. Second, we retrospectively excluded a relative large amount of patients with positive MBI studies due to missing histopathological data. This represents a potential bias of the presented MBI results. However, the excluded cases represented either patients in who the unexpected detection of additional lesions was not clinically relevant (in the sense that it would not have altered the treatment plan), or patients with a relatively low probability of having additional malignant foci (BI-RADS $4 \mathrm{a}$ abnormalities without correlate at targeted US). Third, an injection dose of $600 \mathrm{MBq}$ was applied using a single-head detector. Since others have found comparable results using low-dose protocols (260-500 MBq for singlehead MBI or 150-300 MBq for dual-head MBI) versus highdose protocols [15-17], it would be worthwhile to investigate the performance of the low-dose protocol in the studied patient population, since it could lead to a significantly lower radiation exposure. On the other hand, the strength of this study is that it represents the first series evaluating the additional clinical value of MBI in a large population of patients 
with proven invasive breast cancer scheduled for breastconserving surgery.

\section{Conclusion}

The results of the present study support that MBI is a useful imaging modality, which reveals a high rate of multifocal or multicentric lesions and bilateral disease not visualized by mammography and ultrasound. Additionally, MBI may play an important role in accurate delineation of the tumor extent during preoperative planning. Therefore, the incorporation of this modality to the clinical work-up may lead to better selection of patients who might benefit of BCS. However, larger and prospective studies, preferably using low-dose MBI protocols, are needed to confirm these findings.

\section{Compliance with ethical standards}

Conflicts of interest The authors declare that they have no conflict of interest.

Ethical approval For this type of study formal consent is not required.

Informed consent Informed consent was obtained from all individual participants included in the study.

Open Access This article is distributed under the terms of the Creative Commons Attribution 4.0 International License (http://creativeco mmons.org/licenses/by/4.0/), which permits unrestricted use, distribution, and reproduction in any medium, provided you give appropriate credit to the original author(s) and the source, provide a link to the Creative Commons license, and indicate if changes were made.

\section{References}

1. Netherlands Cancer Registry. http://www.cijfersoverkanker.nl. Accessed 02 Nov 2016

2. International Agency for Research on Cancer (IARC) and World Health Organization (WHO). GLOBOCAN 2012: Estimated cancer incidence, mortality and prevalence worldwide in 2012. http:// globocan.iarc.fr/Pages/fact_sheets_cancer.aspx. Accessed 03 Jan 2018

3. Clough KB, Kaufman GJ, Nos C, Buccimazza I, Sarfati IM (2010) Improving breast cancer surgery: a classification and quadrant per quadrant atlas for oncoplastic surgery. Ann Surg Oncol 17(5):1375-1391

4. Winchester DP, Cox JD (1992) Standards for breast-conservation treatment. CA Cancer J Clin 42(3):134-162

5. Gradishar WJ, Anderson BO, Balassanian R et al (2016) Breast cancer version 2.2016, NCCN Clinical Practice Guidelines in Oncology. http://www.nccn.org/professionals/physician_gls/pdf/ breat.pdf. Accessed 02 Nov 2016

6. Goldsmith SJ, Parsons W, Guiberteau MJ, Stern LH, Lanzkowsky L, Weigert J, Heston TF, Jones E, Buscombe J, Stabin MG, Society of Nuclear Medicine (2010) SNM practice guideline for breast scintigraphy with breast-specific gamma-cameras 1.0. J Nucl Med Technol 38(4):219-224
7. Brem RF, Schoonjans JM, Kieper DA, Majewski S, Goodman S, Civelek C (2002) High-resolution scintimammography: a pilot study. J Nucl Med 43(7):909-915

8. Rhodes DJ, O'Connor MK, Phillips SW, Smith RL, Collins DA (2005) Molecular breast imaging: a new technique using technetium Tc $99 \mathrm{~m}$ scintimammography to detect small tumors of the breast. Mayo Clin Proc 80(1):24-30

9. Jones EA, Phan TD, Blanchard DA, Miley A (2009) Breastspecific gamma-imaging: molecular imaging of the breast using $99 \mathrm{mTc}$-sestamibi and a small-field-of-view gamma-camera. J Nucl Med Technol 37(4):201-205

10. Weinmann AL, Hruska CB, O'Connor MK (2009) Design of optimal collimation for dedicated molecular breast imaging systems. Med Phys 36(3):845-856

11. Hruska CB, Weinmann AL, Tello Skjerseth CM, Wagenaar EM, Conners AL, Tortorelli CL, Maxwell RW, Rhodes DJ, O'Connor MK (2012) Proof of concept for low-dose molecular breast imaging with a dual-head CZT gamma camera. Part II. Evaluation in patients. Med Phys 39(6):3476-3483

12. Mankoff DA, Dunnwald LK, Gralow JR, Ellis GK, Schubert EK, Charlop AW, Tseng J, Rinn KJ, Livingston RB (2002) [Tc$99 \mathrm{~m}]$-sestamibi uptake and washout in locally advanced breast cancer are correlated with tumor blood flow. Nucl Med Biol 29(7):719-727

13. Scopinaro F, Schillaci O, Scarpini M, Mingazzini PL, Di Macio L, Banci M, Danieli R, Zerilli M, Limiti MR, Centi Colella A (1994) Technetium-99 m sestamibi: an indicator of breast cancer invasiveness. Eur J Nucl Med 21(9):984-987

14. Delmon-Moingeon LI, Piwnica-Worms D, Van den Abbeele AD, Holman BL, Davison A, Jones AG (1990) Uptake of the cation hexakis(2-methoxyisobutylisonitrile)-technetium-99 $\mathrm{m}$ by human carcinoma cell lines in vitro. Cancer Res 50(7):2198-2202

15. Brem RF, Ruda RC, Yang JL, Coffey CM, Rapelyea JA (2016) Breast-specific $\gamma$-imaging for the detection of mammographically occult breast cancer in women at increased risk. J Nucl Med 57(5):678-684

16. Kuhn KJ, Rapelyea JA, Torrente J, Teal CB, Brem RF (2016) Comparative diagnostic utility of low-dose breast-specific gamma imaging to current clinical standard. Breast J 22(2):180-188

17. Long Z, Conners AL, Hunt KN, Hruska CB, O'Connor MK (2016) Performance characteristics of dedicated molecular breast imaging systems at low doses. Med Phys 43(6):3062-3070

18. O'Connor MK (2015) Molecular breast imaging: an emerging modality for breast cancer screening. Breast Cancer Manag 4(1):33-40

19. Conners AL, Hruska CB, Tortorelli CL, Maxwell RW, Rhodes DJ, Boughey JC, Berg WA (2012) Lexicon for standardized interpretation of gamma camera molecular breast imaging: observer agreement and diagnostic accuracy. Eur J Nucl Med Mol Imaging 39(6):971-982

20. Spanu A, Sanna D, Chessa F, Manca A, Cottu P, Fancellu A, Nuvoli S, Madeddu G (2012) The clinical impact of breast scintigraphy acquired with a breast specific $\gamma$-camera (BSGC) in the diagnosis of breast cancer: incremental value versus mammography. Int J Oncol 41(2):483-489

21. Kieper DA, Welch BL, Fairchild LH, inventors; Dilon Technologies, Inc., assignee (2012) Gamma guided stereotactic localization system. US Patent US8249693. 21 Aug 2012

22. Collarino A, Valdés Olmos RA, van der Hoeven AF, Pereira Arias-Bouda LM (2016) Methodological aspects of 99mTcsestamibi guided biopsy in breast cancer. Clin Transl Imaging 4(5):367-376

23. Collarino A, Olmos RAV, Neijenhuis PA, den Hartog WC, Smit F, de Geus-Oei LF, Arias-Bouda LMP (2017) First Clinical Experience Using Stereotactic Breast Biopsy Guided by (99m) Tc-Sestamibi. AJR Am J Roentgenol 209(6):1367-1373 
24. Lee A, Chang J, Lim W, Kim BS, Lee JE, Cha ES, Moon BI (2012) Effectiveness of breast-specific gamma imaging (BSGI) for breast cancer in Korea: a comparative study. Breast J 18(5):453-458

25. Killelea BK, Gillego A, Kirstein LJ, Asad J, Shpilko M, Shah A, Feldman S, Boolbol SK (2009) George Peters Award: how does breast-specific gamma imaging affect the management of patients with newly diagnosed breast cancer? Am J Surg 198(4):470-474

26. Zhou M, Johnson N, Gruner S, Ecklund GW, Meunier P, Bryn S, Glissmeyer M, Steinbock K (2009) Clinical utility of breastspecific gamma imaging for evaluating disease extent in the newly diagnosed breast cancer patient. Am J Surg 197(2):159-163

27. Brem RF, Floerke AC, Rapelyea JA, Teal C, Kelly T, Mathur V (2008) Breast-specific gamma imaging as an adjunct imaging modality for the diagnosis of breast cancer. Radiology 247(3):651-657

28. Brem RF, Shahan C, Rapleyea JA, Donnelly CA, Rechtman LR, Kidwell AB, Teal CB, McSwain A, Torrente J (2010) Detection of occult foci of breast cancer using breast-specific gamma imaging in women with one mammographic or clinically suspicious breast lesion. Acad Radiol 17(6):735-743
29. Zhou M, Johnson N, Blanchard D, Bryn S, Nelson J (2008) Realworld application of breast-specific gamma imaging, initial experience at a community breast center and its potential impact on clinical care. Am J Surg 195(5):631-635

30. Sun Y, Wei W, Yang HW, Liu JL (2013) Clinical usefulness of breast-specific gamma imaging as an adjunct modality to mammography for diagnosis of breast cancer: a systemic review and meta-analysis. Eur J Nucl Med Mol Imaging 40(3):450-463

31. Berg WA, Blume JD, Adams AM, Jong RA, Barr RG, Lehrer DE, Pisano ED, Evans WP 3rd, Mahoney MC, Hovanessian Larsen L, Gabrielli GJ, Mendelson EB (2010) Reasons women at elevated risk of breast cancer refuse breast MR imaging screening: aCRIN 6666. Radiology 254(1):79-87

32. Van den Wyngaert T, Strobel K, Kampen WU et al (2016) The EANM practice guidelines for bone scintigraphy. Eur J Nucl Med Mol Imaging 43(9):1723-1738

33. Verberne HJ, Acampa W, Anagnostopoulos C et al (2015) EANM procedural guidelines for radionuclide myocardial perfusion imaging with SPECT and SPECT/CT: 2015 revision. Eur J Nucl Med Mol Imaging 42(12):1929-1940 